\title{
List of notions
}

Ledger

$51 \%$ Attack

6-blocks Confirmation

Altcoins

Artificial Neural

Networks

Bitcoin

Bitcoin/Digital Wallet

Blockchain

Blockchain Fork

Blockchain Mining

Border Gateway

Protocol

Branch Tip
A book, a list, or other collection of financial accounts and/or transactions.

A potential attack on a blockchain network, where a single entity or organization is able to control the majority of the hash power, potentially causing a network disruption. In such a scenario, the attacker would have enough mining power to intentionally exclude or modify the ordering of transactions.

A period of time (commonly around $60 \mathrm{~min}$ ) necessary for adding six new blocks behind a solved block that contain some transaction. After this period, the transaction is considered confirmed because creating a new version of the blockchain that will not contain a given transaction becomes too difficult, because too expensive in computing power.

Cryptocurrencies other than Bitcoin.

A computing system is based on a collection of connected units or nodes called artificial neurons, which loosely model the neurons in a biological brain.

The most popular cryptocurrency, running on a blockchain created in January 2009 by an unknown person under the pseudonym Satoshi Nakamoto.

A software program for holding and trading Bitcoins which uses a person's private key (secret number) to access the person's public Bitcoin address (an identifier of 26-35 alphanumeric characters) and transaction signatures that need to be securely stored; the Bitcoin wallet comes in many forms - the four main types are desktop, mobile, web, and hardware.

A distributed ledger consists of a series of transaction blocks where each block (except the first and the last) is linked with the previous and next block by means of cryptography, making that way an unbreakable chain of blocks.

A situation within the community (of nodes/members of a blockchain P2P network) that occurs when in the network exist two or more versions of the blockchain (i.e., different nodes may have different versions of the file which contains blockchain data).

A peer-to-peer computer process aimed to secure and verify transactions of a given cryptocurrency (such as Bitcoin). Protocol for routing of IP packets (routing protocol); it is a standardized exterior gateway protocol designed to exchange routing and reachability information among autonomous systems on the Internet.

A tip that represents the last individual transaction in a branch tip bundle (see IOTA bundle). 
Byzantine Fault Tolerant System A system that is tolerant (i.e., resistant) to Byzantine Generals Problem; in the strict sense, a BFT system is capable of functioning properly as long as the number of corrupted elements is less than or equal to two-thirds of the overall number of elements.

Byzantine Generals Problem

Car-to-Car Communication

Consensus mechanism

Coordinator (in the context of Tangle)

Cryptocurrency

Cumulative Weight

Decentralized Application

Delay Attack
A class of failures where some of the components/elements of a system may be corrupted in the sense that they have the symptoms (appearance, behavior) that prevent other system's components from reaching agreement (i.e., consensus) among themselves.

Communication that takes place between vehicles with the aim of sharing information that can be used for traffic control or to warn drivers of dangers.

A mechanism (set of rules and protocols) that ensures that all participants dispose of identical copies of the distributed database files (i.e., of the distributed ledger).

A mechanism implemented by IOTA Foundation with the aim to protect Tangle against vulnerabilities such double-spending (as the Tangle is not fully developed, i.e., the overall number of nodes and hash power in the IOTA network are relatively low); the Coordinator mechanism assumes the issuance of a milestone transaction every two minutes.

A digital currency in which encryption techniques are used to regulate the generation of units of currency and verify the transfer of funds, operating independently of a central authority, i.e., of a central bank; most of the cryptocurrencies are based on blockchain technology.

A metric used within the Random Walk algorithm for tip selection in the Tangle; the cumulative weight of a transaction in the Tangle is the total number (plus 1) of all transactions which came after, and at the same time, approved that transaction directly or indirectly. A computer application that runs on a decentralized computing system. DApps have been popularized by distributed ledger technologies such as the Ethereum blockchain; Smart contracts are a key part of DApps.

Attack on the Bitcoin's P2P network that also exploits weaknesses of Border Gateway Protocol (similarly as partition attack); in Delay Attack, the propagation of blocks towards a victim node is effectively slowed down, that is, delayed; the main goal is to keep the victim node uninformed about a new block for almost 20 min, which is achieved through a Man-in-the-Middle attack where attacker modifies messages from victim node to the victim's peer node; as the victim's inquiring messages are wrong (due to modifications performed by an attacker), the victim node is unable to get from the network the freshest solved block (in order to add it on its copy of the blockchain); the victim wastes its computing power and time, instead of using it for solving the next block; consequently, the victim loses its possible earnings. 
Denial of Service Attacks

Difficulty

Difficulty Target

Digital Twin

Directed Acyclic Graph

Distributed Ledger

Double Hash (or Hash-hash)

Algorithm

Double Spending

Problem

Ethash
A cyber-attack that makes unavailable a host (computer, machine, network resource) connected to the Internet; typically accomplished by flooding the targeted host with excessive requests with the aim to overload the system and prevent legitimate requests from being fulfilled.

The algorithm implemented within Bitcoin blockchain that regulates the level of difficulty for solving the PoW task (block solving), so that in average, the frequency of block solving is one block in every 10 min (i.e., 2016 solved blocks for 14 days); as a parameter, it is defined as Difficulty = Difficulty_1_target/ Target_value, showing how many times it is more difficult to find a 256-bit long hash below current target value (determined by parameter Target_value) than to find a hash below a 256-bit reference target value (determined by parameter difficulty_1_target) which has 32 leading zeros. Name of a 32-bit field in the block header that contains parameter Target_value represented via 24-bit mantissa and 8bit exponent; content of this field shown in hexadecimal notation is also known as bits.

A digital replica of potential and actual physical assets (physical twin), processes, people, places, systems, and devices that can be used for various purposes.

A finite directed graph with no directed cycles.

A consensus of replicated, shared, and synchronized digital data geographically spread across multiple sites, countries, or institutions.

Generally, double hashing is a computer programming technique used to resolve hash collisions by using a secondary hash of the key as an offset when a collision occurs; in the Bitcoin blockchain, double-hashing is realized through double use of SHA-256 hashing function, that is, $f(x)=$ sha256(sha256(x)). A potential flaw in a digital cash scheme in which the same single digital token can be spent more than once; it appears if two transactions (one of which is tricky) are sent into the network using the same private key of a fraudulent money sender (a buyer in two different transactions), but sent to different receiving addresses (sellers in two different transactions); as two transactions propagate through the network, one part of the network will accept one transaction and the other part another one.

A PoW mining algorithm implemented by the Ethereum network and Ethereum-based cryptocurrencies; like SHA-3, Ethash is a hash function that belongs to the same (Keccak) family of hash functions, but differs from SHA-3; Ethash was developed with a strong focus on protection from Application Specific Integrated Circuits miners, who were primarily mining Bitcoins. 
Ether

Ethereum Blockchain

Event (in the context of hashgraph)

Famous Witness (in the context of hashgraph)

Fiat currency/Fiat money

Genesis/Genesis Event

Gossip Protocol

Guaranteed (or Secured) Block

Hash Rate/Power

Hash Value

Hashgraph
Native token (cryptocurrency) of the Ethereum blockchain; initially, Ether was not seen as a common digital currency with the main purpose as a means of paying goods and services online, but as an incentive mechanism to power the Ethereum blockchain.

A decentralized open-source blockchain that features smart contract functionality (a project started in 2013 and led by Vitalik Buterin). It is the second-largest cryptocurrency platform by market capitalization (behind Bitcoin).

A data structure created by a node in the hashgraph P2P network at the moment of receiving gossip/synchronization message from another (sending) node; it contains a payload (information about new transactions and other gossips among nodes learned by sending node from the previously received gossip/sync messages), then the timestamp and two hash values that refer to two previous events called self-parent and other-parent event.

A witness of a round that is elected as the famous for that round, in a virtual voting procedure performed by the witnesses of two (or more) upper rounds; it is the witness that most nodes have learned of fairly soon after it was created.

A government-issued currency that is not backed by a commodity such as gold.

The very first transaction in the Tangle/IOTA in which was created the whole amount of IOTA tokens.

A procedure or process of computer $\mathrm{P} 2 \mathrm{P}$ communication based on the way epidemics spread; used by some distributed systems to ensure that data is spread to all members of a group; in some ad-hoc networks, gossip protocol assumes that members forward received data to their neighbors.

A block in a blockchain, after which were added six or more new blocks, it is considered that blockchain forks cannot exist in the blockchain area before the guaranteed block.

The number of hash calculations per second a computing device is capable of executing.

A numeric value of a fixed length that uniquely identifies (a portion of) data, derived as the result of a hash function applied on the data; as the Bitcoin blockchain uses a hash-hash algorithm, i.e., the SHA-256 hash function implemented twice on input data (header of a transaction block), a hash value (of the header of the previous block in the blockchain) written in the block header (of an actual block) is 256 bits long.

A permissioned distributed ledger/platform developed by Leemon Baird (in 2016) and supported by Hedera hashgraph LLC that applies the hashgraph algorithm - a new gossip protocol (logically based on a DAG) that enables a full Byzantine fault-tolerant consensus mechanism; besides native cryptocurrency (the Hedera token), the platform is designed to provide other services such as file storage and smart contracts, i.e., the development of permissionless decentralized applications. 
Hedera Hashgraph

Council

Hedera Hashgraph LLC

Hidden Tip

Industry 4.0

Internet of Things

IOTA Bundle

Lazy Tip

LIDAR

Litecoin

Machine to Machine

Communication
Governing body aiming at supporting the evolution of a stable and decentralized public ledger infrastructure based on the Hashgraph consensus algorithm.

A company that launched the Hashgraph project; provides a public ledger platform for the project's support.

A tip that is not yet seen by a node due to the propagating delay in the IOTA network.

A term used as a meaning for the fourth industrial revolution; as it is not yet completely defined, it rather denotes a set of emerging technologies and tools with a potential to drastically improve various manufacturing processes; it also refers to the synergy between traditional industrial processes and digitalization, whereby the achieved manufacturing solutions are performed through the intelligent networking of machines and processes in the industry with the help of information and communication technology.

Input Transaction (in a bundle) An individual transaction (in a bundle) with the Value field that contains a negative value, meaning that the account specified by the field Address is charged with the amount of IOTAs specified in the Value field.

A system of interrelated computing devices, mechanical and digital machines provided with unique identifiers and the ability to transfer data over a network without requiring human-tohuman or human-to-computer interaction.

A set of several consecutive individual transactions that represent one complete transaction in Tangle, whereby each individual transaction in a bundle directly approves the previous individual transaction, and all together directly approve the same trunk tip - the last individual transaction in another (previous) bundle called trunk tip bundle; first individual transaction in a bundle, besides trunk tip, also directly approves branch tip - the last individual transaction in another (previous) bundle called branch tip bundle.

A tip, i.e., an older transaction in the Tangle selected for approval (by the newest transaction), which had already been approved by other old transactions that came after.

A method for measuring distances (ranging) by illuminating the target with laser light and measuring the reflection with a sensor. A blockchain-based cryptocurrency created and launched by Charlie Lee in 2011; it differs from Bitcoins in aspects like faster block generation rate and use of script algorithm as PoW, which requires much more memory than Bitcoin's hash-hash PoW algorithm; Litecoin is often mentioned as the silver to Bitcoin's gold.

Direct communication between devices using any communications channel, including wired and wireless. 
Man-in-the-Middle

Attack

Master Shard

Merkle Tree

Meta Transaction (in a bundle)

Milestone (in the context of Tangle)

Miner

Mining Pool

Multi-homing

Node

Nonce
An attack where the attacker secretly relays and possibly alters the communications between two parties who believe that they are directly communicating with each other.

Entity (of the Hedera platform) that controls the allocation of nodes to different shards.

A hash-based data structure with tree topology, i.e., a generalization of the hash list in which each leaf node is a hash of a block of data, and each non-leaf node is a hash of its children; Merkle trees typically have a branching factor of 2 , meaning that each node has up to 2 children.

An individual transaction (in a bundle) with the neutral (zero) value written in the field Value; meta transactions are used as extensions of input transactions, usually having the tail parts of the input transactions' signatures.

A transaction in the Tangle issued by the IOTA Foundation as a guarantee that all transactions approved directly or indirectly by this transaction are immediately considered as part of the consensus.

A participant/node in the blockchain's P2P network involved in the blockchain mining process, in which miners try to solve a computationally intensive task (typically a mathematical problem or puzzle) in order to gain the opportunity to add a new block of transactions to the blockchain, and as a reward, to earn a certain amount of cryptocurrency (defined by the applied blockchain algorithm), as well as to get the transactions' fees.

A joint group of cryptocurrency miners, who combine their computational resources over a network in order to solve a PoW task and add a block of transactions to the blockchain; if the pool does the PoW successfully, it is rewarded with cryptocurrency tokens; the reward is divided to the miners who contributed according to the proportion of each miner's processing power or work relative to the whole group.

The practice of connecting a host or a computer network to more than one network. Most commonly, it is done to increase reliability or performance.

A participant in the $\mathrm{P} 2 \mathrm{P}$ network of a particular blockchain on which is run the blockchain's native coin/cryptocurrency or decentralized application.

Abbreviation for the number only used once that blockchain miners are solving, i.e., searching for, before adding a new block to the blockchain; it is a 32-bit value put in the header of a transaction block (of the Bitcoin blockchain) which meets the difficulty level restrictions in the sense that the 256-bit hash value of the block header (with the nonce included) is small enough, i.e., that has a required number of leading binary zeros; for each block, the hash value of the block header (containing 
Other-parent Event

Output Transaction (in a bundle)

Partition Attack

Peer-to-Peer

network

Permissioned DL

Permissionless DL

Proof of Capacity

Proof of Space

Proof of Stake
The last event created earlier by the hashgraph node, which sent a new gossip/sync message (to the node, which then creates a new event).

An individual transaction (in a bundle) with the Value field that contains a positive value, which represents the amount of IOTAs that is to be added to the account specified by the field Address. A type of attack on the Bitcoin's P2P network in which an adversary (e.g., a malicious transit autonomous system on the Internet) exploits vulnerabilities of Border Gateway Protocol and performs prefix hijacking of a part of the P2P network, i.e., isolates a group of victim nodes (of the Bitcoin's P2P network) in a certain point of the Internet; the consequence is that all blocks (and transactions contained within) solved during isolation period by the victim nodes will be discarded after the end of isolation because, after that, the blockchain version maintained on the victim nodes cannot survive in the whole network (due to smaller hash power of victim nodes compared to the rest of the network).

A network of equally privileged nodes (peers) connected by means of direct links, all of them participating in a decentralized application.

A distributed ledger in which each network participant (node) needs permission from a central entity (e.g., owner or administrator of the ledger) to access the network and make changes, i.e., to add transactions to the ledger.

A distributed ledger without a central owner that controls network access, so any participant may join the network and add transactions to the ledger if the participant (computer node) runs the relevant software that complies with the rules of the applied consensus mechanism.

See Proof of Space

Also called Proof of Storage or Proof of Capacity; the concept originally introduced for showing that one has a legitimate interest in service by allocating a sufficient amount of memory or disk space to solve a challenge presented by the service provider; it can be used as consensus mechanism as well, for achieving distributed consensus among the members/nodes of a cryptocurrency blockchain P2P network.

A type of consensus mechanism, i.e., an algorithm for achieving distributed consensus (among the members/nodes of a cryptocurrency blockchain P2P network) where the winner, i.e., the creator of the next transaction block (that is to be added to the blockchain), is chosen among the blockchain community members in dependence of the members' stakes (the amounts of cryptocurrency members posses). 
Proof of Work

Random Walk Monte Carlo

Algorithm

Round (in the context of

hashgraph)

Round Created (of an event)

Round Received (of an event)

Seeing (in the context of hashgraph)

Segregated Witness Protocol

Self-parent Event

Shards/Sharding

Architecture

Site (in the context of Tangle)

Smart Car
A type of consensus mechanism, i.e., an algorithm for achieving distributed consensus (among the members/nodes of a cryptocurrency blockchain P2P network) that requires some work from the service requester, usually meaning processing time by a computer while solving a given mathematical problem, a computationally intensive puzzle, etc.

Algorithm for selection of a tip that will be approved by a new transaction that should be added to the Tangle.

A part (a portion of events) that the hashgraph diagram is divided into as it grows in time.

A serial number of the round an event belongs to in the hashgraph diagram; round created of an event is calculated as follows: if the event strongly sees a supermajority of witnesses of round $r$, where $r$ is the max of the round created of the event's parents, then the round created of the event is $r+1$ (otherwise, round created of the event is $r$ ).

A serial number of the first round after an event (in the hashgraph diagram) which fulfills the condition that all the famous witnesses of that round see this event (not necessarily strongly); in other words, if an event is seen by all round $\mathrm{n}$ famous witnesses, this event is said to be received (by the community of nodes) in round $n$, i.e., that it has round received of $n$.

A situation (in the hashgraph diagram) when an event $Y$ is linked with some previous event $X$ via at least one path no matter through how many nodes the path passes.

A protocol upgrade of the Bitcoin blockchain that enables protection from transaction malleability and increases the block size limit (from $1 \mathrm{MB}$ to a little under $4 \mathrm{MB}$ ) on the blockchain by removing signature data from Bitcoin transactions.

The previous event created by the hashgraph node, which receives a new gossip/sync message (and creates a new event). The architecture (i.e., the arrangement) of the Hedera platform nodes that should enable scalability and continuous decentralization of the platform in the future; the architecture assumes the existence of shards (partitions, groups) of nodes, where each shard contains a relatively small number of nodes. Graph representation of a transaction in Tangle (issued by a node of IOTA network).

In the past, this term denoted a car with advanced electronics; now (and in a stricter sense), a smart car is the car that, besides numerous enhancements, also possesses a property to be highly interconnected (e.g., via WiFi, Bluetooth, 4G/5G) and with a certain level of self-driving automation capabilities (from assisted driving to the fully autonomous driving). 
Smart Contract

Smart Factory

Smart Manufacturing

Solidified Block

Solidity (programming

language)

Strongly Seeing (in the context of hashgraph)

Supermajority (in the context of hashgraph)

Sybil Attack

Tangle/IOTA

Target_value

Tip

TLS Encryption

Transaction
Computer code running on a DLT platform; it executes automatically when specific conditions are met; due to the execution on a DLT platform, a smart contract runs exactly as programmed (according to the agreed contract terms), i.e., there is no chance of being changed, interrupted, censored or interfered by any third party; smart contract execution enables the exchange of value.

A highly digitalized and connected environment where machinery and equipment are able to improve processes through automation and self-optimization.

This term is practically synonymous with Industry 4.0, which gives solutions involving one or more technologies such are: Internet of Things, Artificial Intelligence, Augmented Reality, Automation/Robotics, or Additive manufacturing/3D printing. A block in a blockchain, after which were added a few (i.e., less than 6) new blocks.

Programming language for smart contracts; initially developed for the execution of smart contracts on the Ethereum platform. A situation (in the hashgraph diagram) when an event $Y$ is linked with some previous event $X$ in a way that all paths that connect $X$ and $Y$ contain (in whole) a supermajority of nodes (including the nodes that created events $X$ and $Y$ ).

Any group of hashgraph nodes that gathers more than $2 / 3$ of the total number of nodes.

Type of attack where attacker subverts the reputation system of network service by creating a large number of pseudonymous identities and uses them to gain a disproportionately large influence; named after the subject of the book Sybil, a case study of a woman diagnosed with a dissociative identity disorder. A distributed ledger project that logically relies on a new DAG structure (called Tangle), designed to record and execute transactions between machines and devices in the IoT ecosystem, realizing its native token (cryptocurrency) called IOTA.

A parameter that represents the upper limit under which the 256 bit hash value should be found for successful verification of a new block.

A (relatively new) transaction previously added to the Tangle, which is selected to be directly approved by a new transaction. Abbreviation from Transport Layer Security - a cryptographic protocol designed to provide communications security (primarily privacy and data integrity) between two or more communicating computer applications over a network; TLS operates on top of Transmission Control Protocol.

A sequence of information exchange between communication partners. 
Transaction block

Transaction Fee (or Miner Fee)

Tritt

Trunk Tip

Trustless Truth

Tryte

Vehicle-to-Environment (or Vehicle-to-Everything) Communication

Virtual Voting (in the context of hashgraph)

Vote Weighting

Witness of a Round (in the context of hashgraph)
A block (i.e., set) of transactions - the basic data structure/unit which is linked with other blocks in a series by means of cryptography, creating that way a blockchain.

The fee paid (in cryptocurrency) by the purchaser within a transaction to a miner who verified this transaction (i.e., who added the transaction onto blockchain); with Bitcoin blockchain, a miner who successfully adds a block of transactions to the blockchain gets the fees for all transactions in the block. A digit in balanced ternary logic; can take values from set $\{+1$, $0,-1\}$.

A tip that represents the last individual transaction in a trunk tip bundle (see IOTA bundle).

A term that denotes the state of a community in which exists a general consensus about something (e.g., about the trust in Bitcoin), where no one trusts anyone and where (just because of overall distrust) a set of rules (or a mechanism like PoW) is imposed to all participants, achieving in that way that eventually, everyone is confident about some common thing of interest.

A word of 3 Tritts (in balanced ternary logic); for designation of Trytes in IOTA (27 different values) are used 26 uppercase letters "A", "B", "C", . . ., "X", "Y", "Z" and symbol "9" (which in balanced ternary logic represents the value +1 ).

Passing of information from a vehicle to any entity that may affect the vehicle, and vice versa; it is a vehicular communication system that incorporates other more specific types of communication as Vehicle-to-Infrastructure, Vehicle-to-Network, Vehicle-to-Vehicle, Vehicle-to-Pedestrian, Vehicle-to-Device and Vehicle-to-Grid.

A voting process in which the community (i.e., the nodes of hashgraph P2P network) collectively and democratically establishes the consensus on the transactions' order in time; it is called virtual because nodes do not really send their votes over the Internet (i.e., the Yes/No decisions regarding the so-called famous witnesses), but each node calculates what votes other nodes would have sent, based on its knowledge of what other nodes know.

An adjustment of virtual voting algorithm realized with the aim to enable usage of the algorithm in the community (of members/ hashgraph nodes) whose members are not equal and where the significance of a member depends on the stake (the amount of Hedera's native tokens) the member possesses; the adjustment is made through simple redefinitions of notions of majority, supermajority, and median value, whereby the members' stakes are taken into account.

An event (at the beginning of a round) that strongly sees a supermajority of witnesses from the previous round; witnesses of round 1 are the first events ever created by the corresponding nodes. 